patients with secondary KLS, 5 had stroke or brain trauma, and the remainder had various diseases, including genetic, infectious encephalitis, and multiple sclerosis. Symptoms in secondary cases began later than in primary KLS, and the disease course was longer and more severe. (Arnulf I, Zeitzer JM, File J et al. Kleine-Levin syndrome: a systematic review of 186 cases in the literature. Brain December 2005;128:2763-2776). (Respond: Dr I Arnulf, Federation des pathologie du Sommeil, Hopital Pitie-Salpetriere, 47-83 Boulevard de l'Hopital, 75013 Paris, France).

COMMENT. First named KLS by Critchley et al (1942), these authors emphasized the male preponderance, adolescent onset of episodic hypersomnia, compulsive eating, and spontaneous recovery. The infectious factor in causation of KLS requires further study, especially in relation to viral or post-infectious autoimmune encephalitis, with involvement of the hypothalamus. Neuropathology in 2 primary KLS cases showed inflammatory changes in the thalamus, hypothalamus, and basal ganglia. A genetic factor is suggested, since onesixth of patients were Jewish. Epilepsy and depressive causes have been ruled out.

\title{
CORTICOSTEROIDS IN HERPES SIMPLEX VIRUS ENCEPHALITIS
}

A non-randomized retrospective study of 45 adult patients (12 young adults) with herpes simplex virus encephalitis (HSVE), treated with acyclovir alone (23 patients) and acyclovir plus corticosteroids (22 patients), was conducted at Nihon University School of Medicine, Tokyo, Japan. At 3 months, a poor outcome was found in older patients, those with lower Glasgow Coma Scores at initiation of therapy, and in those not receiving corticosteroids in the acute stage. (Kamei S, Sekizawa T, Shiota $\mathrm{H}$ et al. J Neurol Neurosurg Psychiatry Nov 2005;76:1544-1549). (Respond: Dr S Kamei, Division of Neurology, Department of Medicine, Nihon University School of Medicine, 30-1 Oyaguchikamimachi, Itabashi-ku, Tokyo 173-8610, Japan).

COMMENT. This study suggests that a combination of acyclovir with corticosteroids initiated at the acute stage of HSVE is a predictor of a better prognosis than acyclovir alone. The beneficial effects of corticosteroids may be explained by reduction of brain edema and regulation of the host immune response, decreasing the extent of viral infection.

Openshaw $\mathrm{H}$ and Cantin EM, in an editorial commentary ( $\mathbf{J}$ Neurol Neurosurg Psychiatry Nov 2005;76:1469), opine that the effectiveness of coriicosteroids in HSVE can only be determined by prospective, randomized studies. The benefits observed in the above retrospective study could be related to selection of cases and to the corticosteroid therapy per se.

Concerns that early corticosteroid administration may increase viral CNS spread, leading to a restriction of steroids to cases with significant brain edema, may not be justified, based on recent animal studies cited by Kamei et al (Thompson KA et al. Herpes simplex replication and dissemination is not increased by corticosteroid treatment in a rat model of focal herpes encephalitis. J Neurovirol 2000;6:25-32; Meyding-Lamade UK et al. Experimental HSVE: a combination therapy of acyclovir and glucocorticoids reduces longterm MRI abnormalities. J Neurovirol 2003;9:118-125). Early diagnosis is a factor in prognosis, and a characteristic EEG multifocal periodic pattern may be helpful when CT and ultrasound are normal in neonatal HSVE (Mikati MA et al. Neurology 1990;40:1433-37). 